\title{
Diagnosing the level of Glaucoma from Fundus Image Using Empirical Wavelet Transform
}

\author{
NarayanaSwamy R ${ }^{1}$, Syed Thouheed Ahmed ${ }^{2 *}$,Thanuja $\mathrm{K}^{3}$, Ashwini $\mathrm{S}^{4}$, Syeda Aye- \\ sha Siddiqha ${ }^{4}$, AfifaSalsabil Fathima ${ }^{4}$ \\ ${ }^{1}$ JAIN University, Bengaluru \\ ${ }^{1}$ Dr. T. Thimmaiah Institute of Technology, KGF \\ ${ }^{3}$ REVA University, Bengaluru \\ ${ }^{2}$ Cambridge Institute of Technology, Bengaluru \\ syed.edu.in@gmail.com
}

\begin{abstract}
An increased pressure of fluid in optic nerve can subsequently leads to permanent blindness are known as Glaucoma. The normal pressure of eye is $15 \mathrm{mmHg}$ or even lower, once it is higher than $30 \mathrm{mmHg}$ then there is risk in vision loss. There are many existing technique that require experienced clinicians and cost effective. These systems use higher order spectra and discrete wavelet transform features for extracting the values and fed to classifier for normalization and ranking the feature. In this paper presenting a new methodology for diagnosis of glaucoma based on EWT. Empirical wavelet transform is applied on image to format the sub band which is also called as decomposed image. These features are sustained into neural network system that produces ne value from $n$ iteration and classify images into mild, intermediate and heavily affected eye using Fundus images.
\end{abstract}

Keywords:Empirical Wavelet Transform (EWT), Glaucoma detection, Image processing.

\section{Introduction}

Eyes are the important sense organ for vision which detects the surrounding light and convert the light into electrochemical impulse. An eye indicates the health of entire human body. One of the most common irreversible neurodegenerative [1] disease caused in eye is glaucoma. The condition where improper drain of fluid in eye causing high pressure leading to intraocular disorder ultimately resulting in degenerated nerve optic nerve hence glaucoma is also called as "noiseless hoodlum of sight"[5], age beyond 40 years, diabetics, high blood pressure, high use of steroid, eyes tumors and eye wounds are some of main causes.

The existing system for diagnosis of glaucoma uses higher order spectra where the features are extracted and fed to classifier to select best kernel [4] function for automated decision. But the segmentation has failure in certain standard like threshold, boundary fixing. They are not able to capture the curve and edges of image[2]. The 
main drawback is that the images are decomposed into many R,G,B, and gray scale samples of images into various components resulting in high computation time and added noise. These techniques are cost effective and require experienced clinicians. It may also cause some unavoidable or unexpected error.

In proposing method, diagnosis at low rate with respect to empirical wavelet transform (EWT) is performed. The images are decomposed into various frequency bands such as normal, intermediate affected and glaucoma affected, where it reduces the workload clinicians. The Fundus image obtain will focus only on decomposition and extracting of morphological features such as optic disk space in eye also called as cup to optic disk ratio[3]. Continuously iterating the value to obtain high accuracy. The performance of system does not depend on the result of image.

\section{Literature Survey}

\subsection{Empirical Wavelet Transform}

Wavelet transform is an infinity set of different transforms dependency $\mathrm{n}$ function used for their computation. It uses the function that is localized in real space and Fourier space. Discrete wavelet can decomposed single mutually in set of wavelets that is constructed from scaling function. Continues wavelet transform compute single value based on scale wavelet. The drawback of wavelets are discontinues and irregularity of signal causing low efficient in computing intensive signals. The 2D wavelets uses classifier kernel function [3] decompose image into various components like R, G, B, Gray scale components in form of variation of pixel intensities.Empirical wavelet transform (EWT) is an adaptive method in determining the independent signal that doesn't have any predefined function. The idea behind EWT is to decomposed image or signal into various sub signals based on wavelet frames [4]. The decomposed component can reduce noise based on local window maxima [5],by continuously iterating the value using neural network concept, wherein the input is iterated for several times the result of first iterating is again iterated to produce single result. The diagnosis between normal eye and glaucoma eye is based on cup to optic disk ratio called cupping [3]. The EWT has more consistent decomposition combining the advantages of Fourier analysis and wavelet transform to extract different modes in complicated signal essentially design a set of suitable wavelet filters to get different bands of signals. The EWT output is composed on filtering with scaling function and wavelets. The careful selection of wavelet [7][8][9] and decomposed level in order to obtained in good time frequency resolution. A similar approach is recorded with color image processing by authors in [6][10]. 


\section{$3 \quad$ Methodology}

The optic nerve transmit image from retina to brain enabling to detect surrounding light. The main components of retina are optic disk, optic cup, and neuro-retinal rim. The relationship between optic disk and optic cup is cup to disk ratio. The space present in front of eye is called anterior chamber, aqueous humor is a clear fluid that flows in and out the chamber thereby nourishing the surrounded tissues. This fluid is drained through a spongy meshwork called trabecular meshwork. Due to some unexpected reasons if the meshwork ends up plainly blocked the weight inside eye increase resulting in structural change of nerve fiber and optic disk parameter like diameter volume and area which thins the neuro-retinal rim also, referred as cupping.

\subsection{System Architecture}

The input is given in form of images, such as normal, intermediate, and glaucoma affected eye. These images are decomposed into various bands with respect to time. It continually iterates the value based on segments, the obtain value is further evaluated to fixed pixel and threshold value. If the given image value is greater than threshold value then it is normal or else intermediate and lesser value is affected eye. (Fig.1)

\subsection{Mathematical Modeling}

Decomposition. The given image is decomposed into various tiny segments with respect to time; these segments are Fourier transform designed as series of wavelet filters.

Step 1: Differentiating the image with respect to time is given as $\frac{\partial(\mathrm{img})}{\partial(t)}$ where $t$ is segments

In general $T=\sum_{i=0}^{n} \frac{\partial(\mathrm{img})_{i}}{\partial(\mathrm{t})_{i}}$ whereiistimeforonesliceof segments

Step 2: The decomposed image $T_{A}$ for $\mathrm{n}$ slice of time is given as $T_{A}=\sum_{j=0}^{n}\left\{\sum_{i=0}^{n} T_{i}\right\}_{j}$ 


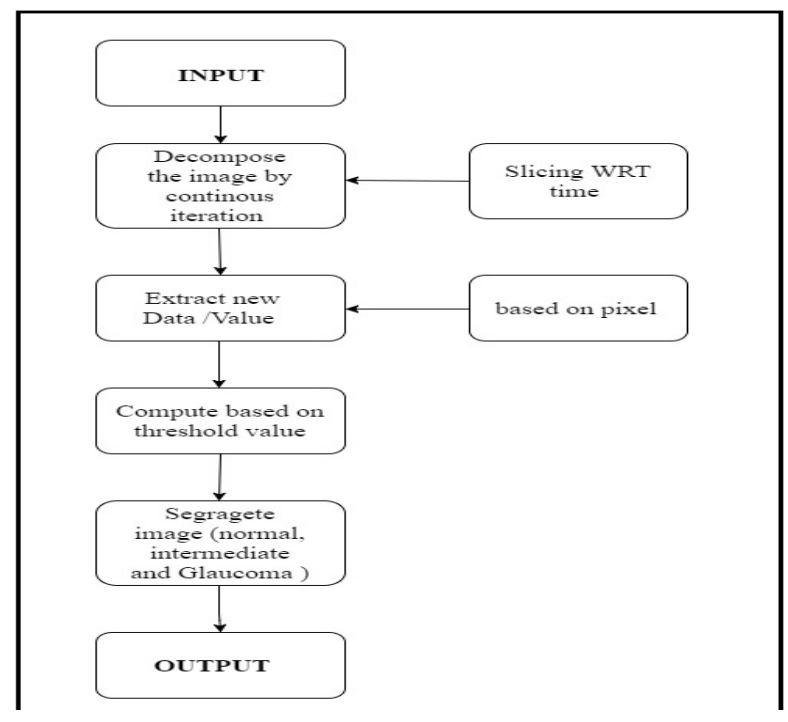

Fig.1. System Architecture for proposed approach

Step 3: Finally, image decomposition can be given as, $T_{A}=\left[\sum_{j=0}^{n} \sum_{i=0}^{n}\left(T_{i}\right)_{j}\right]$

Value extraction: obtaining the data /information from decomposed image. The existing Fourier transform obtained the boundary using rows and columns of an image. The proposed EWT obtains the boundary by slicing the image based on pixel value.

Step 4: The pixel value is given as $\mathrm{N}$ so taking either Row or Column pixel value can be estimated $T_{A}=\frac{1}{N_{R W}} \sum_{j=0}^{n}\left\{\sum_{i=0}^{n}\left(T_{i}\right)\right\}_{j}$

Step 5: Expanding above equation for further simplification

$$
T_{A}=\frac{1}{N_{R W}} \sum_{j=0}^{n}\left[\sum_{i=0}^{n} \frac{\partial\left(T_{i}\right)_{j}}{\partial(t)}\right]
$$

Algorithm computation. The image is computed based on threshold value if the value is graeter then normal eye else affected by glaucoma

Step 6: For each decomposed image $T_{A}$ with respect to each time $\forall T_{i} \Rightarrow T_{A}$ for each

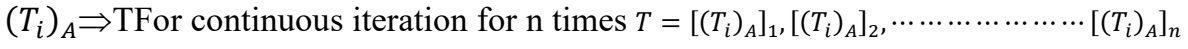

Step 7: In general, $T=\frac{\sum_{j=0}^{n}\left[\left(T_{i}\right)_{A}\right]_{j}}{n}$ segregating positive and negative images. 


\section{$4 \quad$ Results and Discussions}

In proposed methodology EWT are extracted from decomposed components, this feature effectively captured by variations of pixels and yields high accuracy. Glaucoma is characterized by regular damage of retinal nerve fiber. Various morphological features are obtained from Fundus images and fed to neural network concept, wavelet based features are extracted using different wavelet families to achieve accuracy.

From Fig 2 the input (fig a) is the Fundus image of eye, when given to EWT the input is being filtered (fig b) for future extraction except the optic disk remaining area is been darken with black producing black producing black and white image (fig c). The white space in black background is the optic disk which is being produced (fig d). Later the processed optic disk is taken as input to continuous detection (fig e). Again the entire Fundus image is taken as gray scale detection (fig f). Only the optic disk region is segmented from gray scale (fig g). The segmented part is divided into various tiny segments of pixels (fig h). The segmented region of optic disk is again filtered to obtain the affected area (fig i). Again the background is detected only for segmented region of optic disk (fig j). A boundary is been detected for that region (fig $\mathrm{k}$ ). The detected boundary is been smoothened (fig 1). The skeleton structure is extracted from smoothened image boundary (fig $\mathrm{m}$ ). Finally the extracted value of area, diameter and volume is been compared with given threshold value (fig n). Resulting in output such as mild affected, intermediate affected and heavily affected.

Fig $(0)$ gives the performance graph between mean of squared error vs. number of iteration taken to extract the value. (fig $p$ ) gives the training state gradient value and validation value. (fig q) gives regression graph for output vs. number of iteration where it reaches to saturated point in graph.

\section{Conclusion}

In this paper, the proposed method for diagnosis of glaucoma level. This is done by using EWT where the Fundus image are decomposed into various tiny pixel and optic disk is being segmented for identify the presence of glaucoma. The decomposed components produces linearity for regression that is effectively captures for variation in pixels. The methodology can also be extended to diagnosis glaucoma at an early stage. The features are computed based on the texture of decomposed components of different frequency bands. 


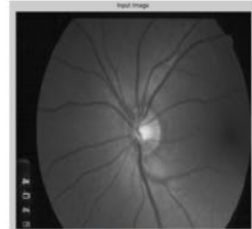

(a)

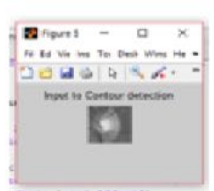

(e)

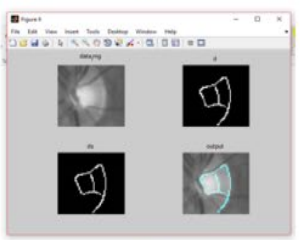

(i)

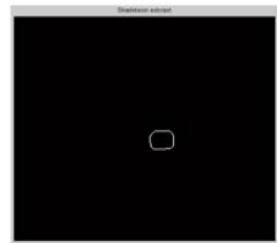

(m)

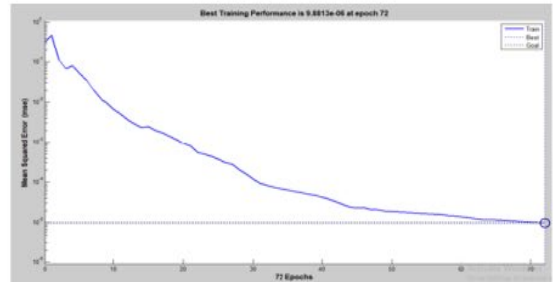

(o)

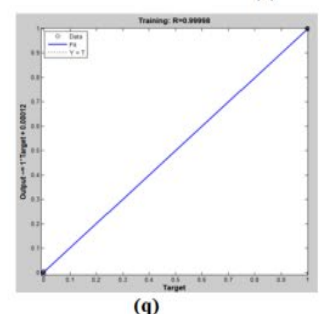

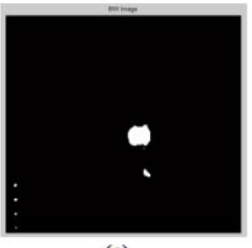

(c)

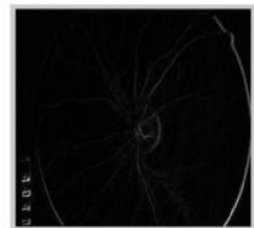

(f)

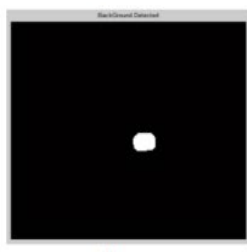

(j)

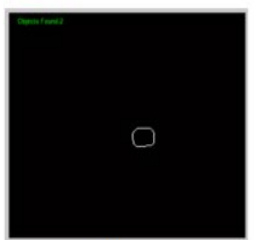

(n)

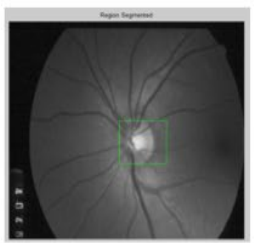

(g)

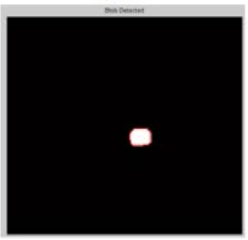

(k)

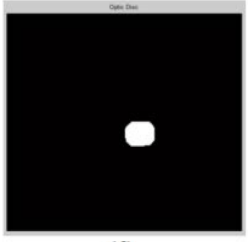

(d)

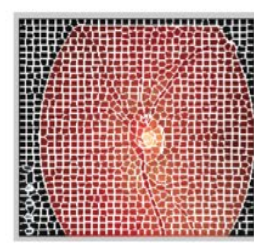

(h)

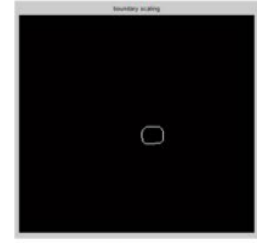

(I)

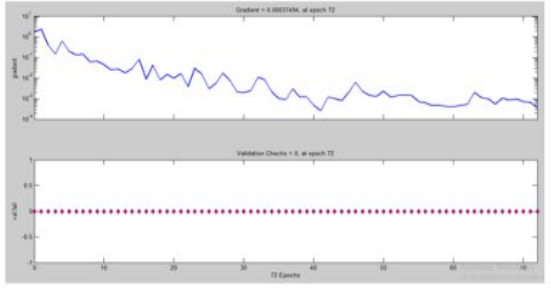

(p)

Fig.2.Diagnosis mild effect of glaucoma 


\section{References}

1. Future trend forecst by empirical wavelet transform and auto regressive moving average ,, qiushengwang, haipeng Li and chunxiazhang

2. Automated Diagnosis of Glaucoma Using Empirical Wavelet Transform and Correntropy Features Extracted from Fundus Images ShishirMaheshwari, Ram BilasPachori, and U. RajendraAcharya

3. Wavelet-Based Energy Features for Glaucomatous Image Classification SumeetDua, Senior Member, IEEE, U. RajendraAcharya, PradeepChowriappa, Member, IEEE, and S. VinithaSree

4. Gunashree, M., Ahmed, S. T., Sindhuja, M., Bhumika, P., Anusha, B., \&Ishwarya, B. (2020). A New Approach of Multilevel Unsupervised Clustering for Detecting Replication Level in Large Image Set. Procedia Computer Science, 171, 1624-1633. https://doi.org/10.1016/j.procs.2020.04.174

5. S. T. Ahmed, "A study on multi objective optimal clustering techniques for medical datasets," 2017 International Conference on Intelligent Computing and Control Systems (ICICCS), Madurai, 2017, pp. 174-177, doi: 10.1109/ICCONS.2017.8250704.

6. K. D. Singh and S. T. Ahmed, "Systematic Linear Word String Recognition and Evaluation Technique," 2020 International Conference on Communication and Signal Processing (ICCSP), Chennai, India, 2020, pp. 0545-0548, doi: 10.1109/ICCSP48568.2020.9182044

7. S. T. Ahmed, H. K. Priyanka, S. Attar and A. Patted, "Cataract density ratio analysis under color image processing approach," 2017 International Conference on Intelligent Computing and Control Systems (ICICCS), Madurai, 2017, pp. 178-180, doi: 10.1109/ICCONS.2017.8250705.

8. Dafni Rose J, Vijayakumar K, "Data Transmission Using Multiple Medium Concurrently”, IJET,2018.

9. K. Vijayakumar, S. Suchitra and P. Swathi Shri, "A secured cloud storage auditing with empirical outsourcing of key updates", Int. J. Reasoning-based Intelligent Systems, Vol. 11, No. 2, 2019.

10. K. Vijayakumar, Chokkalingam Arun, "Integrated cloud-based risk assessment model for continuous integration", Int. J. Reasoning-based Intelligent Systems", Vol. 10, Nos. 3/4, 2018. 